\title{
Swiss Quality Award 2016: Die Gewinner (1)
}

\section{Nadja Jenni ${ }^{a}$, Fabienne Hohlb}

${ }^{a}$ Wissenschaftliche Mitarbeiterin der Abteilung Daten, Demographie und Qualität der FMH; ${ }^{b}$ Abteilung Kommunikation der FMH

Zum siebten Mal wurde am 1. Juni 2016 der Swiss Quality Award während des Nationalen Symposiums für Qualitätsmanagement im Gesundheitswesen verliehen. Der Preis würdigt praxiserprobte und zukunftsweisende Qualitätsprojekte und ist mit 22 Patronatspartnern im Gesundheitswesen breit abgestützt*.

Für den Swiss Quality Award 2016 wählte die interdisziplinär zusammengesetzte Jury von Qualitätsexpertinnen und -experten aus beinahe 40 eingereichten Projekten die Gewinner in den Preiskategorien «ambulant», «stationär» und "sektorübergreifend». Jede Kategorie ist mit 10000 Franken dotiert. Zudem kürte eine weitere fachkompetente Jury das beste Projektposter mit dem Swiss Quality Poster-Award, der mit 2000 Franken ausgezeichnet wird.

Doch nicht nur die Gewinner profitieren vom Swiss Quality Award, denn nach der Preisverleihung werden die Projektposter der Gewinner und sämtlicher am Swiss Quality Poster-Award teilnehmenden Teams auf www.swissqualityaward.ch aufgeschaltet. Die OnlinePublikation ermöglicht allen Interessierten Einblick in die besten Qualitätsprojekte und bietet Fachpersonen eine ideale Anregung für vertiefte Diskussionen.

Die drei Trägerorganisationen des Swiss Quality Award, das Center of Excellence for Medical Registries and Data Linkage (Swiss RDL)/Institut für Sozial- und Präventivmedizin der Universität Bern, die Schweizerische Gesellschaft für Qualitätsmanagement im Gesundheitswesen sQmh und die Verbindung der Schweizer Ärztinnen und Ärzte FMH gratulieren den Gewinnerinnen und Gewinnern herzlich! Und freuen sich, in dieser und der nächsten Ausgabe der Schweizerischen Ärztezeitung die preisgekrönten Qualitätsprojekte vorzustellen.

Weitere Informationen zum Swiss Quality Award finden Sie unter www.swissqualityaward.ch oder schreiben Sie uns ein E-Mail an info[at]swissqualityaward.ch.

\section{Die Tanzstation - (kosten-)effektive Sturzprävention für Senioren}

\section{Friederike Schulte ${ }^{a}$, Andrea Dinevskib \\ a Dr. sc. ETH; ${ }^{b}$ lic. phil.}

Stürze im Alter sind für die Betroffenen einschneidend und für unser Gesundheitssystem belastend. Mit schweizweit jährlich 1,36 Mrd. Franken kostet ab Alter 65 jeder Sturz mit Verletzungsfolge umgerechnet 16730 Franken [1]. Besonders gehäuft treten Stürze in Institutionen auf; 70\% aller Seniorenheimbewohner stürzen mindestens einmal pro Jahr.

Tanzen ist als Sturzprävention seit langem anerkannt. Weil Musik positive Gefühle auslöst, spricht Tanzen auch weniger bewegungsfreudige Senioren an. In der Forschung wurden sogenannte Tanzstationen unter-

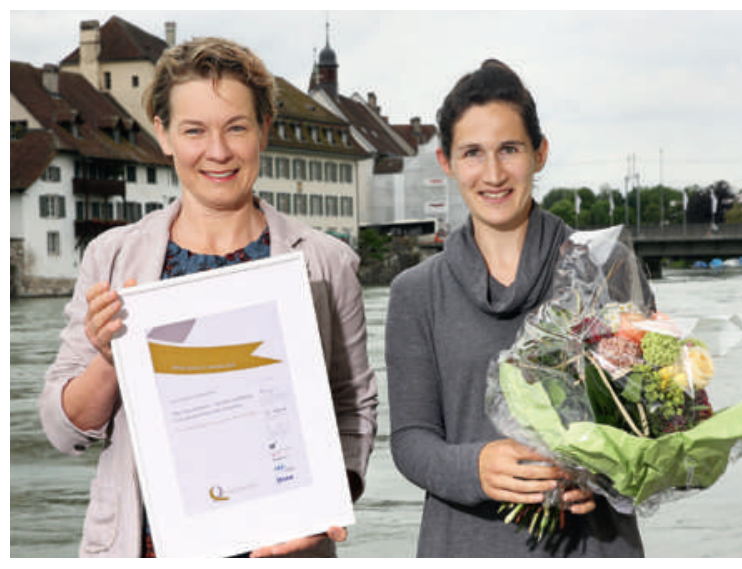

Die Gewinner in der Kategorie "ambulant», Andrea Dinevski und Frederike Schulte. 


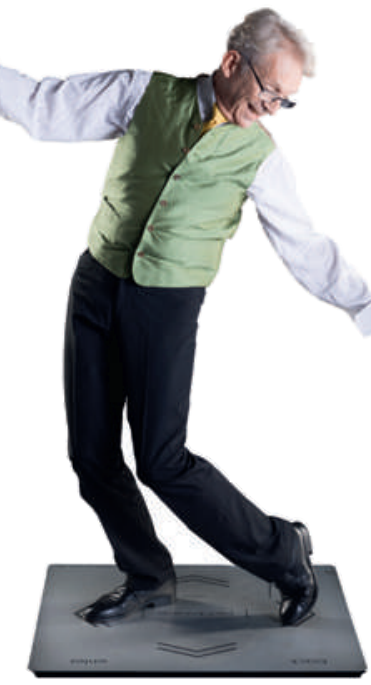

Tanzen auf der Tanzstation fördert Balance und Reaktion.

\section{Korrespondenz:} Andrea Dinevsk REDANCE GmbH Steinwiesstrasse 32 8032 Zürich andrea.dinevski[at] redance.ch sucht; die Akzeptanz dieser Trainingsform wird mit $87 \%$, ihre sturzreduzierende Wirkung mit 58\% angegeben. Bis heute waren keine den Bedürfnissen von Senioren entsprechenden Tanzsysteme erhältlich. REDANCE hat diese Lücke geschlossen und Seniorenzentren, Physiotherapien und anderen Institutionen eine wissenschaftlich etablierte Methode mit Gute-Laune-Faktor zur Sturzprävention zugänglich gemacht.

Dies sieht konkret so aus: Die tanzende Person steht auf einer Bodenplatte (siehe Abb.) und hört OriginalMusik der 40er, 50er oder 60er Jahre. Ein Fernseher zeigt dem Tanzenden die kommenden Schritte an, die im Takt der Musik auf die Platte zu setzen sind. Die Schwierigkeitsstufen sind wähl- und der Tanzerfolg ist messbar. Die Rückmeldung in Echtzeit fördert den Wunsch, mehr und noch besser zu tanzen. Pilotversuche in Seniorenresidenzen zeigen eine hohe Akzeptanz und stossen auf Begeisterung. In einer eigenen Studie mit 20 Probanden im Privatbereich wurden
Verbesserungen im Treppenabwärtssteigen, in der Reaktionsfähigkeit und in der Balance festgestellt. Nach 3 Monaten tanzten noch 92\% der Studienteilnehmer. Eine weitere Zielgruppe darf im neuro-rehabilitativen Bereich vermutet werden. Demenz- und ParkinsonPatienten profitieren von Tanztherapien. Studien bei Demenzpatienten belegen einen Anstieg des MiniMental-Status-Tests um 21\% und bei Parkinson Verbesserungen im Gleichgewichtsverhalten.

Abgesehen vom Wohl der Senioren ist eine Tanzstation ab der Verhinderung von einem Sturz mit Verletzungsfolge im Jahr rentabel. Gesamthaft könnten mit einer Sturzreduzierung um 58\% jedes Jahr 789 Mio. Franken an Gesundheitskosten eingespart werden. Die Bereitstellung einer Tanzstation als Sturzpräventionsmassnahme leistet einen wesentlichen Beitrag zur Verbesserung der Patientensicherheit im ambulanten Bereich.

Literatur

1 bfu-Report Nr. 71 (2015)

\section{Weniger Bluttransfusionen dank eines Patient-Blood-Management-Programms mit Monitoring und Feedback}

\author{
Tarun Mehraa , Donat R. Spahn ${ }^{b}$ \\ ${ }^{a}$ Dr. med.; ${ }^{b}$ Prof. Dr. med., FRCA
}

Bluttransfusionen sind für Patienten nicht risikofrei [1] und zudem kostenintensiv. Patient Blood Management (PBM) ist ein umfassendes Konzept, welches eine präemptive Reduktion des Bedarfs an allogenen Blutprodukten anstrebt, mit dem Ziel einer höheren Patientensicherheit und eines verbesserten Outcomes. Eine tragende Säule des PBM-Konzepts ist die Einführung restriktiver Transfusions-Trigger [2] im Rahmen von anerkannten und akzeptierten Transfusionsrichtlinien. Trotz deren Einführung 2012 sank die Zahl der Transfusionen am UniversitätsSpital Zürich nicht. Erst das PBM-Monitoring-und-Feedback-Programm, welches in enger Abstimmung mit den Klinikdirektoren und in Zusammenarbeit mit der IT umgesetzt wurde, reduzierte die Anzahl an Bluttransfusionen pro $1000 \mathrm{~Pa}-$ tienten innerhalb eines Jahres um über $25 \%$, wodurch

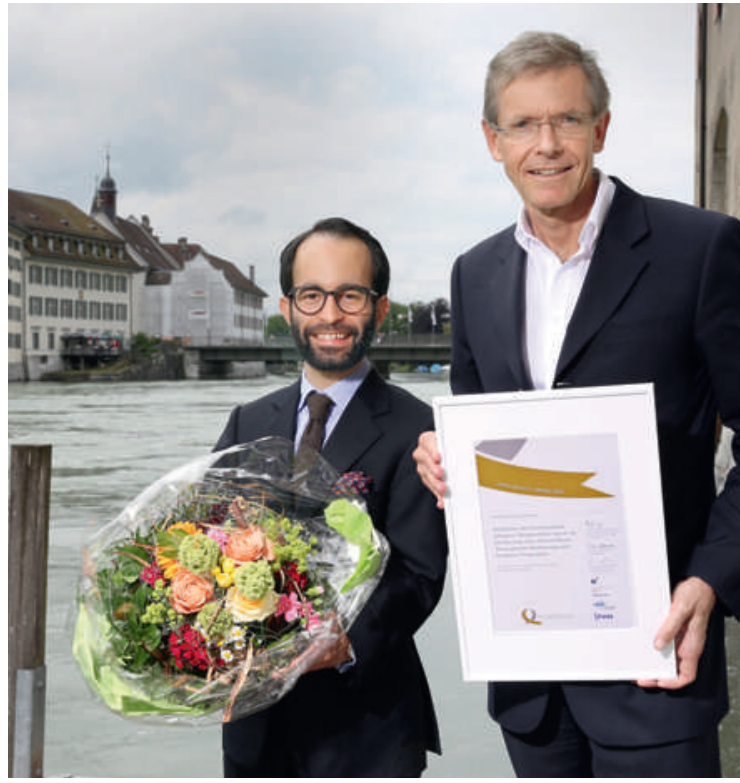

Die Gewinner in der Kategorie «stationär», Dr. Tarun Mehra und Prof. Dr. Donat Spahn. 


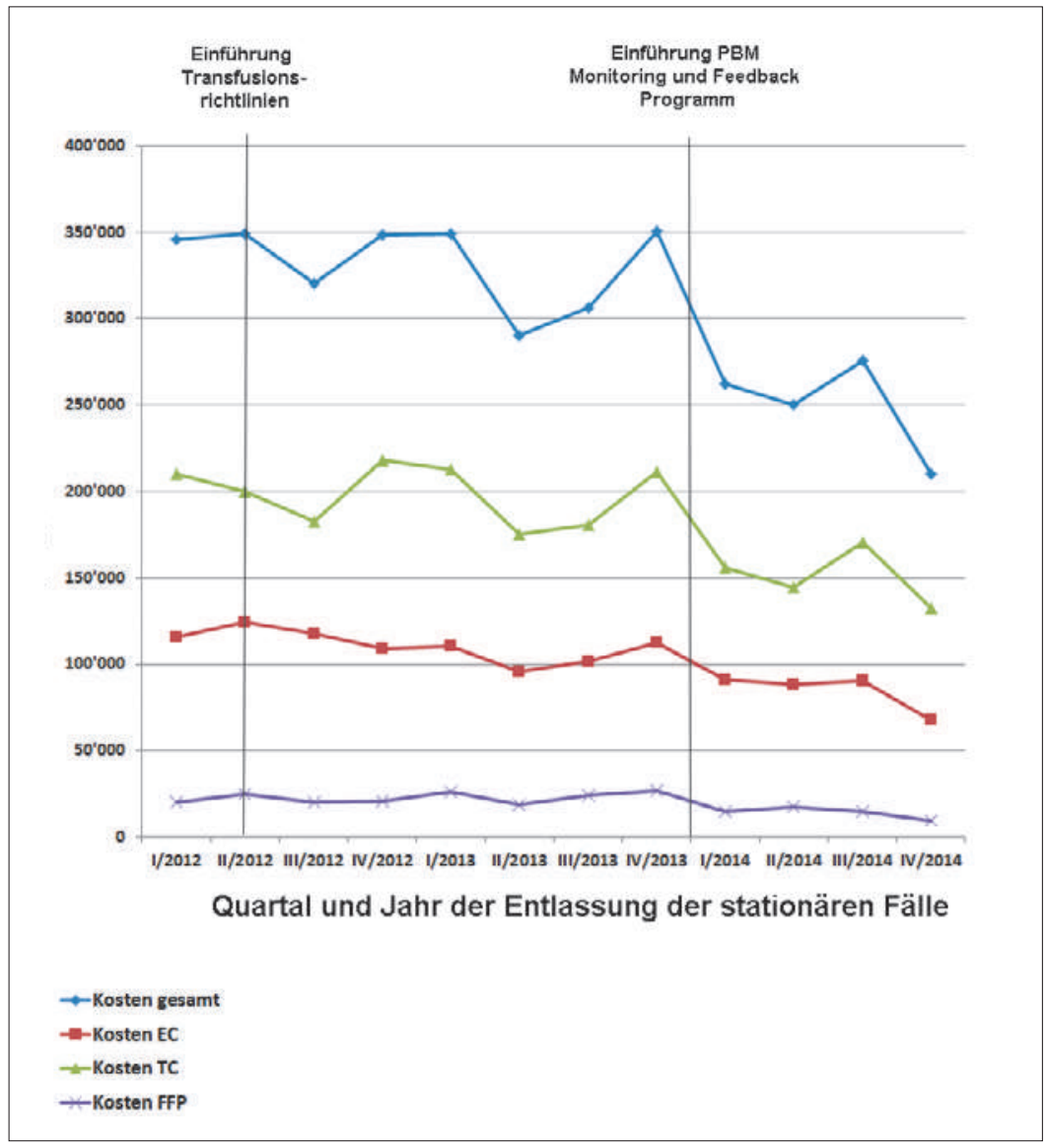

Trend der Kosten für Transfusionen pro 1000 stationäre Fälle, vor und nach der Einführung vom Patient-Blood-Monitoring-Programm mit Monitoring und Feedback.

\section{Korrespondenz:}

Prof. Dr.

Donat R. Spahn, MD, FRCA,

Professor und Klinik-

direktor, Institut

für Anästhesie

Universität und

UniversitätsSpital Zürich,

CH-8091 Zürich,

donat.spahn[at]usz.ch sich Ausgaben von ca. 2 Millionen CHF vermeiden liessen [3].

Hierzu wurde jede Verordnung einer Transfusion elektronisch erfasst. Die Transfusionen wurden quartalsweise bezogen auf den letzten, der Transfusionsverordnung vorausgehenden Laborwert ausgewertet, im Rahmen einer überparteilichen Kommissionssitzung analysiert, diskutiert und die Ergebnisse den Kliniken im Rahmen eines Reportings übermittelt.

Das Projekt ist ein gutes Beispiel dafür, dass erst die Einführung einer transparenten Ergebnismessung und deren Diskussion in einem kollegialen Umfeld die Umsetzung gemeinsam beschlossener Leitlinien sicherstellt. Monitoring-und-Feedback-Programme sind auch in anderen Bereichen wie z.B. Thromboseprophylaxe und Medikamentensicherheit denkbar. Die Ausweitung solcher Programme auf andere Bereiche und andere Spitäler könnte die Patientensicherheit auf nationaler Ebene massgeblich verbessern.

\section{Literatur}

1 Spahn DR, Goodnough LT. Alternatives to blood transfusion. Lancet 2013;381: 1855-65.

2 Carson JL, Carless PA, Hebert PC. Transfusion thresholds and other strategies for guiding allogeneic red blood cell transfusion. Cochrane Database Syst Rev 2012;4: CDOO2042.

3 Mehra T, Seifert B, Bravo-Reiter S, Wanner G, Dutkowski P, Holubec $\mathrm{T}$ et al. Implementation of a patient blood management monitoring and feedback program significantly reduces transfusions and costs. Transfusion. 2015;55:2807-15.

* Die Patronatspartner des Swiss Quality Award 2016 sind: Bundesamt für Gesundheit BAG, Die Spitäler der Schweiz H+, CURAVIVA Schweiz, Nationaler Verein für Qualitätsentwicklung in Spitälern und Kliniken ANQ, Qualitätsmedizin Schweiz, Schweizerische Akademie der medizinischen Wissenschaften SAMW, Schweizerischer Berufsverband der Pflegefachfrauen und Pflegefachmänner SBK, foederatio medicorum chirurgicorum helvetica fmCh, Föderation Schweizer Psychologinnen und Psychologen FSP, Schweizerischer Verband Medizinischer Praxis-Assistentinnen SVA, ErgotherapeutInnen-Verband Schweiz evs, Schweizerischer Hebammenverband SHV, Schweizerischer Verband der Berufsorganisationen im Gesundheitswesen SVBG, Spitex Verband Schweiz, Medizinaltarif-Kommission UVG (MTK), pharmaSuisse, physioswiss, Curafutura, santésuisse, Schweizerische Stiftung SPO Patientenschutz, Dachverband Schweizerischer Patientenstellen, Patientensicherheit Schweiz.

\section{Bildnachweise}

Foto Seite 868 und Seite 869 unten rechts: Swiss Quality Award Innovations in Healthcare (Lenka Reichelt)

Foto Seite 869 oben links: redance

Grafik Seite 870: Gem. Autoren, UniversitätsSpital Zürich 